\title{
Joint Antenna Diversity and Combined DFE/Decoding of Trellis Coded Modulation on Frequency-Selective Fading Mobile Radio Channels
}

\author{
Guillem Femenias, Antoni Gelonch and Josep Lluís Gàlvèz \\ Dept. de Teoria del Senyal i Comunicacions \\ Universitat Politècnica de Catalunya (UPC) \\ Apdo. 30.002, 08080 Barcelona, SPAIN
}

\begin{abstract}
In this paper a Monte Carlo simulation study of a combined FDMA/TDMA Slow Frequency-Hopping Trellis Coded adaptive receiver for multipath Rayleigh fading channels is presented. The receiver structure, first proposed by Proakis and then developed by Ralf Mehlan and Heinrich Meyr for use on HF fading channels, comprises Decision Feedback Equalizer (DFE), block deinterleaver and TCM decoder in one unit. Antenna diversity is considered leading to a joint diversity and combined equalization/decoding scheme. Furthermore, Cyclic Slow Frequency Hopping is used to attain intrinsic frequency diversity to perform a near ideal channel interleaving with a short processing delay. In order to asses the perforinance of this scheme Monte-Carlo simulations of the system have been carried out where the dynamic behavior of the mobile radio channel has been simulated by means of a selective equal-energy two-ray Rayleigh model, with a variable delay, $\tau$, between the two rays. Bit error rate (BER) is obtained as a function of signal-to-noiseratio (SNR) and with $T$, Doppler spread, number of frequencies in the frequency-hopping pattern, number of DFE coefficients and TDMA frame lenght as parameters.
\end{abstract}

\section{INTRODUCTION}

Many radio communication channels such as $\mathrm{HF}$ shortwave ionospheric, UHF tropospheric scatter (beyond-the-horizon), VHF ionospheric forward scatter, UHF land mobile radio, and others can be effectively modelled as frequency-selective time-variant multipath fading channels [1], [2]. Frequency solectivity causes intersymbol interference (ISI), time variation calls for an adaptive receiver and multipath fading results in a very low SNR when channel exhibits a deep fade.

It is well known that the optimum receiver for a signal impaired by ISI and additive white noise is a whitened matched filter (WMF) followed by a symbol rate sampler and a maximum likelihood sequence estimator (MLSE) [3]. However, due to the computational complexity of this type of receiver, which grows exponentially with the lenght of the channel memory and the size of the data symbol alphabet, suboptimal reduced computation detection algorithms such as decision feedback equalizer [4], reduced state maximum likelihood sequence estimation [5], [6], M-algorithm MLSE equalizers [7], and others, have been developed.
Trellis coded modulation (TCM), although originally developed for telephone channels [8], has received also considerable interest in the field of mobile radio [9]-[12]. The primary advantage of TCM over modulation schemes employing traditional error correction coding is its ability to achieve increased power efficiency without the customary expansion of bandwidth introduced by the coding process. Furthermore, when combined with interleaving of sufficient depth, TCM is known to provide some form of time diversity that can be used to improve the reliability of a multipath-fading channel over conventional uncoded modulation. In addition to using coding, multipath fading can be also combated by using antenna diversity reception where the receiver is provided with multiple independently faded replicas of the same information. Diversity is effective because it diminishes the probability of simultaneously having two or more independently faded channels in a deep fade [2].

Joint equalization/decoding on a super trellis as proposed in [13], [14] is not feasible for multipath-fading channels due to the use of the interleaving/deinterleaving proces to break up the memory of the channel, which is in contrast to the equalization task where the channel memory is exploited. Soft output equalizers [15], [16], [17], which generate reliability information for each data symbol that once passed through the deinterleaver enables the decoder to operate in a soft decision mode, are limited to channels with short ISI profiles, because of their rapid growth in complexity with increasing lenght of the channel impulse response (CIR). In this paper we follow a different approach, first proposed by Proakis [1] and then developed by Ralf Mehlan and Heinrich Meyr [18] for use on HF fading channels, in which the receiver structure comprises Decision Feedback Equalizer (DFE), block deinterleaver and TCM decoder in one unit. Furthermore, we consider the use of antenna diversity, leading to a joint diversity and combined equalization/decoding scheme. A combined FDMA/TDMA Cyclic Slow Frequency Hopping scheme, similar to that adopted by pan-European GSM or DECT systems, is assumed. Known training symbols of each TDMA time slot are exploited in the decoding process and Cyclic Slow Frequency Hopping is used to attain intrinsic 
frequency diversity to perform a near ideal channel interleaving with a short processing delay. In order to asses the performance of this scheme Monte-Carlo simulations of the system have been carried out where the dynamic behavior of the mobile radio channel has been simulated by means of a selective equal-energy two-ray Rayleigh model, with a variable delay, $\tau$, between the two rays. Bit error rate (BER) is obtained as a function of signal-to-noise ratio (SNR) and with $\tau$, Doppler spread, number of frequencies in the frequency-hopping pattern, number of DFE coefficients and TDMA frame lenght as parameters.

\section{SYSTEM MODEL}

In the transmitter, input bits (representing data or digitally encoded speech) are fed into a trellis encoder. The complex-valued output symbol stream is next block interleaved (buffer matrix with $N R$ rows and NC columns) to randomize the distribution of symbols that could be affected by amplitude fades of duration greater than one symbol period and to make full use of trellis code properties. Both the base and the mobile stations transmit a sequence of message bursts, located in the assigned TDMA time slots. The transmitted burst structure includes a preamble, a block interleaver row of $N C$ information symbols and a guard time. The preamble, known at the receiver, allows an estimate of the channel impulse response (CIR) and a proper initialization of the receiver parameters, on a burst-by-burst basis. Once filtered by the shapping filter, $h_{T}(t)$, these symbols provide the complex baseband transmitted signal $s(t)$. Finally, to make use of the FH intrinsic frequency diversity, the $N R$ formed slots are modulated according to the assigned frequency-hopping pattern. By setting the frequency-hopping separation sufficiently large, a null correlation among the different frequency channels can be assured [2].

Since $K$-diversity reception is used in the system, the channel is modeled as $K$ independent dispersive fading channels corrupted by additive white Gaussian noise. At the receiver side, each of the $K$ diversity branches consists of an FH linear demodulator followed by a matched filter, used to get the maximum signal-to-noise power ratio at the sampling instant, and a noise whitening filter, employed to whiten the colored noise coming out of the matched filter and to make the overall channel impulse response of the channel minimum phase or nearly so (this can be controlled by adapting whitening matched filter (WMF) coefficients to channel variations). Complex-valued symbol streams at the output of the $K$ whitening matched filters are fed to the diversity combiner. Finally, these symbols are stored into the block deinterleaver in successive rows and read out in columns for joint equalization/decoding.

\section{A. Optimal diversity combiner}

Let $S_{k}, R_{k}$ and $N_{k}$ be the $k$-component row vectors whose $i$-th components are $s_{i}, r_{i}$ and $n_{i}$, respectively, for $i=1,2, \ldots, k . S_{k}$ represents the block interleaved transmitted sequence, $R_{k}$ the received sequence and $N_{k}$ the sequence of complex zero-mean additive Gaussian noise components with single-sided power spectrum density equal to $N_{0}$. Assuming, in general, that we are receiving a signal transmitted onto the $j$-th hopping frequency $(j=1,2, \ldots, N R)$, the complex-valued sample at the output of the whitening matched filter, at time $t=i T$, can be expressed as

$$
r_{j, i}=\sum_{h=0}^{8-1} s_{i-h}^{A} y_{j, i, h}+n_{i}
$$

where the superscript $\Delta$ is a "wild card" for " $T$ " and " $D$ " (training or data) and the sequence of complex values given by the vector

$$
Y_{j, i}=\left[\begin{array}{llll}
y_{j, i, 0} & y_{j, i, 1} & \cdots & y_{j, i, 8-1}
\end{array}\right]
$$

represents the causal equivalent sampled impulse response at time $t=i T$ of the linear baseband channel, being the cascade of the channel impulse response $\boldsymbol{H}_{j, l}$ (formed by the modulator pulse shaping and $j$-th mobile radio-link propagation selective distortions) and the corresponding WMF $W_{j, l}$. Optimal diversity combining is performed by adding the $K$ weighted WMF output signals $r_{j, 1}$,

$$
r_{i}=\sum_{j=1}^{K} p_{j, i} r_{j, i}
$$

where the combining weights can be determined from the instantaneous channel energies $\left(E_{h}\right)_{j, l}=\left\|H_{j, l}\right\|^{2}$ as

$$
p_{j, i}=\frac{\left(E_{h}\right)_{j, i}}{\left(E_{k}\right)_{i}}
$$

with

$$
\left(E_{h}\right)_{i}=\sum_{k=1}^{K}\left(E_{h}\right)_{k, i} .
$$

Therefore, expression (3) can be rewritten as

$$
r_{l}=\sum_{h=0}^{g-1} s_{i-h}^{\Delta} f_{i, h}+\eta_{i}
$$

where $\eta_{1}$ is a sample of a complex zero-mean additive Gaussian noise with single-sided power spectrum density equal to $N_{0} /\left(\mathrm{E}_{\mathrm{h}}\right)_{i}$ and the sequence of complex values given 
by the vector $F_{i}=\left[\begin{array}{llll}f_{i, o} & f_{i, 1} & \ldots & f_{i,-1}\end{array}\right]$ represents the sampled equivalent channel impulse response of the combined signal and is equal to the weighted sum of all diversity channel impulse responses.

\section{B. Combined Equalization and Decoding}

As shown in Fig. 2, symbols at the output of the optimal diversity combiner are fed into the block deinterleaver matrix in successive rows and read out in columns for joint equalization/decoding. From (6) it can be seen that the intersymbol interference (ISI) in each received symbol can be described in terms of the symbols in $g-1$ previous columns. Therefore, all ISI terms corrupting the received symbols in the first data column will depend on known training symbols and estimated channel impulse response. Thus, in this case, corrected symbols can be computed as,

$$
u_{i}=r_{i}-\sum_{h=1}^{8-1} s_{i-h}^{T} f_{i, h}-s_{i}^{D}+\eta_{i}
$$

With the assumption that the additive noise is white and Gausian distributed, the conditional probability for one corrected symbol is given by

$$
p\left(u_{i} \mid s_{i}^{D}\right)=\frac{1}{\left(\pi N_{0} /\left(E_{k}\right)_{i}\right)^{1 / 2}} \exp \left\{-\frac{\left(E_{h}\right)_{i}\left|u_{i}-s_{i}^{D}\right|^{2}}{N_{0}}\right\}
$$

where the complex-valued component $\hat{s}_{i}^{D}$ represents a possible transmitted signal at time $t=i T$. Thus, maximum-likelihood branch metrics for soft Viterbi trellis decoding will be given by,

$$
m\left(\hat{s}_{i}^{D}, x_{i} ;\left(E_{h}\right)_{i}\right)=-\left(E_{h}\right)_{i}\left|\hat{s}_{i}^{D}-x_{i}\right|^{2}
$$

Corrected symbols of the first data column are fed into the soft Viterbi decoder for MLSE on the code trellis. After a suitable decoding delay $\delta$ which depends on the constraint lenght of the code the decoder outputs a final decision $\hat{s}^{D}{ }_{i-\delta}$. Choosing the number of rows in the interleaver and deinterleaver matrices to be equal to $\delta+1$, the decoder will output the first decoded symbol corresponding to the information contained in a particular deinterleaving matrix, when all received data of the first row had been processed. This decoded symbol could be used for ISI cancellation of the first symbol in the second column of the matrix. This corrected symbol will be fed into the Viterbi decoder that will decode the second channel symbol. And so on, until last symbol of current data block is corrected and fed into the trellis decoder. Computation of branch metrics of the code trellis is affected by previous decisions, thus, the system will be subject to error propagation.

Notice that the algorithm requires adaptive estimation and deinterleaving of the complete complex channel impulse response for each symbol interval. Thus, the complexity and the amount of memory necessary to store this information grow rapidly with the lenght of the channel impulse response and the size of interleaving matrix. To reduce the complexity of the algorithm, the complex channel impulse response will be estimated only during the short training periods and between these probes it will be held constant.

\section{SIMULATION RESULTS AND CONCLUSIONS}

Computer simulation programs have been set up in order to evaluate the bit error rate (BER) performance of joint antenna diversity and combined DFE/decoding of TCM on frequency-selective fading mobile radio channels. The dynamic behavior of the channel has been simulated by means of a simple two-ray model, with a variable delay $\tau$ between the two rays. Both the direct and delayed signal components have been independently Rayleigh faded, with equal average power, and frequency shifted according to a Doppler power spectrum related to the given vehicle speed [2]. Pulse shaping has been a square-root raised-cosine with rolloff parameter equal to 0.5 . The ideal equivalent channel impulse response has been taken for calculating the instantaneous channel energy, adjusting the matched filter coefficents and computing the DFE coefficients on a burst-by-burst basis. The minimum mean square error (MMSE) criterion has been adopted to calculate the DFE coefficients. DFE forward coefficients coincide with those of the whitening filter and DFE feedback coefficients have been used to correct the received symbols. Interleaving and deinterleaving matrices of 20 rows and a variable number of columns have been assumed. In order to investigate multi-level quadrature amplitude modulation (MQAM) schemes, the selected trellis code has been the rate $(2 \times 1 / 2)$, $2 \times 16$ states, 2x4PAM (16QAM) code, designed by M.L. Moher and J.H. Lodge [12]. This code offers a time diversity order equal to 5 , which is the maximum time diversity of all trellis codes having the 16QAM symbol constellation.

Simulation results assuming an ideal training process are presented in Figs. 1-6. Fig. 1 shows the bit error rate for $K$ equal to one and two, for a two-ray model with a normalized delay between the two rays $\tau / T=0.5$, a frequency hopping pattern with 20 frequencies, a TDMA time slot with 30 data symbols, a DFE with $6+6$ coefficients (forward + feedback coefficients) and with the signal to noise ratio and normalized Doppler spread $f_{d} T$ as parameters. Normalized fading rates of $2.0 \cdot 10^{-4}, 4.0 \cdot 10^{-4}$ 
and $8.0 \cdot 10^{-4}$ have been considered. It can be seen that the system performance deteriorates as the normalized fading rate increases and an average bit error floor is clearly shown for fast Rayleigh fading. Nevertheless, comparing the irreducible error probability obtained without diversity with that obtained with two antenna diversity, we can conclude that space diversity applies to the irreducible error floor as well.

Fig. 2 presents the average bit error rate performance of the system for a normalized Doppler spread $f_{d} T=4.0 \cdot 10^{-4}$, a frequency hopping pattern with 20 frequencies, a TDMA time slot with 30 data symbols, a DFE with $6+6$ coefficients and with $E_{b} / N_{0}$ and $\tau / T$ as parameters. With $\tau / T=0.0$, the channel model is equal to a flat Rayleigh channel. The performance improvement obtained by increasing $\tau / T$ to 0.5 shows that a time diversity benefit is gained by using the combined DFE decoding scheme. As long as the predicted CIR closely approximates the actual CIR, this can be achieved. With increasing echo delays, this condition can only be satisfied by increasing the number of DFE coefficients. The effectiveness of the proposed receiver in fighting long echo delays is investigated in Figs. 3 and 4 where BER versus $\tau / T$ is reported for given $E_{b} / N_{0}$ values and with the number of DFE coefficients as parameter. As it can be seen, the range in which the time diversity gain is maximum depends on the number of DFE coefficients. For a DFE with $4+4$ coefficients the time diversity gain is maximum around $\tau / T=0.5$, while for $\tau / T>1.5$, a significant degradation is observed. However, for a DFE with $12+12$ coefficients this range expands beyond $\tau / T=4.0$. All the above results have been obtained assuming a normalized Doppler spread $f_{d} T=4.0 \cdot 10^{-4}$, a frequency hopping pattern with 20 frequencies and a TDMA time slot with 30 data symbols.

In Figs. 5 and 6 the effects resulting from the unavailability of sufficient number of frequencies in the frequency hopping pattern are shown. It can be seen that for a frequency hopping pattern with fifteen frequencies, the results practically coincide with those obtained for a pattern of twenty frequencies. Even with only ten hopping frequencies, this degradation does not exceed $1.5 \mathrm{~dB}$ for average bit error rates in the range of interest. It is worthy of point out that, with a normalized Doppler spread $f_{d} T=4.0 \cdot 10^{-4}$, the case of a frequency hopping pattern with only one frequency corresponds approximately to a transmission system without interleaving.

In summary, the simulation results demonstrate the robust performance of the joint antenna diversity and combined DFE/decoding of trellis coded modulation on frequency selective fading mobile radio channels. In the future, further simulations must be performed in order to asses the performance of the system using a real channel impulse response estimator. Furthermore, bit error rate (BER) has to be obtained as a function of signal-to-noise ratio (SNR) and with decoding decision delay, TDMA frame lenght, correlation coefficient between the diversity branches, ... as parameters.

\section{REFERENCES}

[1] J. G. Proakis, Digital Communications. Singapore: McGraw-Hill, 1989.

[2] W.C. Jakes, ed., Microwave mobile communications. New York: John Wiley and Sons, 1974.

[3] G. D. Forney, Jr., "Maximum-likelihood sequence estimation of digital sequences in the presence of intersymbol interference," IEEE Trans. Inf. Theory, vol. IT-18, pp. 363-378, May 1972.

[4] S.U.H. Qureshi, "Adaptive equalization," IEEE Proceedings, vol. 73, pp. 1349-1387, Sep. 1985.

[5] M.V. Eyuboglu and S.U.H. Qureshi, "Reduced-state sequence eatimation with set partitioning and decision feedback," IEEE Trans. on Comm., vol. 36, pp. 13-20, Jan. 1988.

[6] A.P. Clark, Adaptive detectors for digital modems. London: Pentech Press, 1989.

[7] A. Baier and G. Heinrich, "Performance of M-algorithm MLSE equalizers in frequency selective fading mobile radio channels," in Proc. of ICC, pp. 281-285, 1989.

[8] G. Ungerboeck, "Channel Coding with Multilevel/Multiphase Signals," IEEE Trans. Inf. Theory, vol. 28, pp. 5-25, Jan. 1982.

[9] D. Divsalar and M. K. Simon, "Trellis coded modulation for $4800-9600 \mathrm{bits} / \mathrm{s}$ transmission over a fading mobile satellite channel", IEEE Joumal Sel. Areas Commun., vol. SAC-5, pp. $162-175,1987$.

[10] M. K. Simon and D. Divsalar, The performance of trellis coded multilevel DPSK on a fading mobile satellite channel", IEEE Trans. Vehic. Tech., vol. 37, pp. 78-91, 1988.

[11] J. Cavers and P. Ho, "Analysis of the error performance of trellis-coded modulations in Rayleigh fading channels, "IEEE Trans. Commun. vol. 40, pp. 74-83, Jan. 1992.

[12] M.L. Moher and J.H. Lodge, "TCMP-A modulation and coding strategy for rician fading channels," IEEE Joumal Sel. Areas Commun., vol. 7, pp. 1347-1355, Dec. 1989.

[13] M.V. Eyuboglu and S.U.H. Qureshi, "Reduced-state sequence estimation for coded modulation on intersymbol interference channels, " IEEE Joumal Sel. Areas in Comm., vol. 7, pp. 989-995, August 1989.

[14] P.R. Chevillat and E. Eleftheriou, "Decoding of trellis-encoded signals in the presence of intersymbol interference and noise, "IEEE Trans. on Comm., vol. 37, pp. 669-676, July 1989.

[15] P. Hoeher, "TCM on frequency-selective fading channels: a comparison of soft-output probabilistic equalizers, "in Proc. of IEEE Globecom, pp. 401 .4.1-401.4.6, 1990.

[16] W. Koch and A. Baier, "Optimum and Suboptimum detection of coded data disturbed by time-varying intersymbol interference," in Proc. of ICC, pp. 1679-1684, 1990.

[17] R. Mehlan, J. Wittkopp and H. Meyr, "Soft output MAP equalization and trellis coded modulation for severe frequency selective fading channels," in Proc. of ICC, pp. 331.4.1-331.4.5, 1992.

[18] R. Mehlan and H. Meyr, "Combined equalization/decoding of trellis coded modulation on frequency-selective fading channels, " in Coded modulation and bandwidth-efficient transmission, E. Biglieri and $\mathbf{M}$. Luise (eds). Tirrenia, Italy: Elsevier Science Publishers B.V., 1992. 


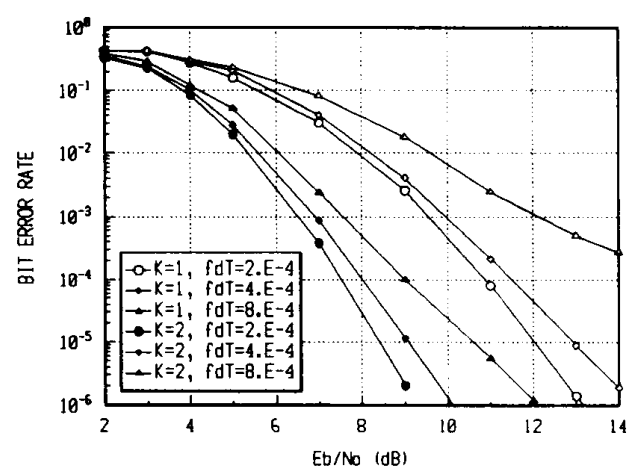

Fig. 1. BER performance for $K$ equal to one and two with $f_{d} T$ as parameter. $(\tau / T=.5$, DFE $(6+6), 20$

hopping frequencies)

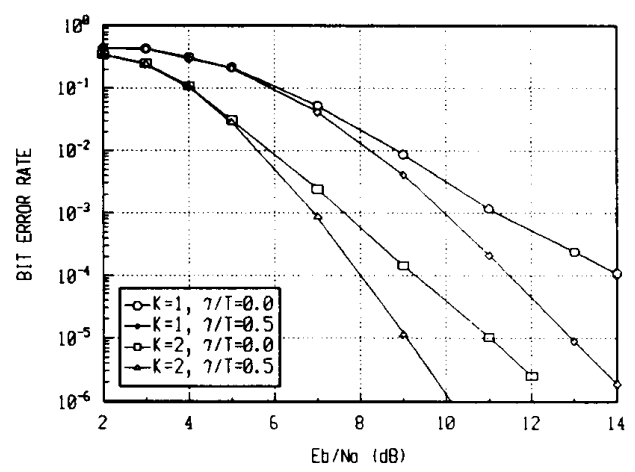

Fig. 2. BER performance for $K$ equal to one and two with $\tau / T$ as parameter. $\left(f_{d} T=4.0 \cdot 10^{-4}\right.$, DFE $(6+6), 20$ hopping frequencies)

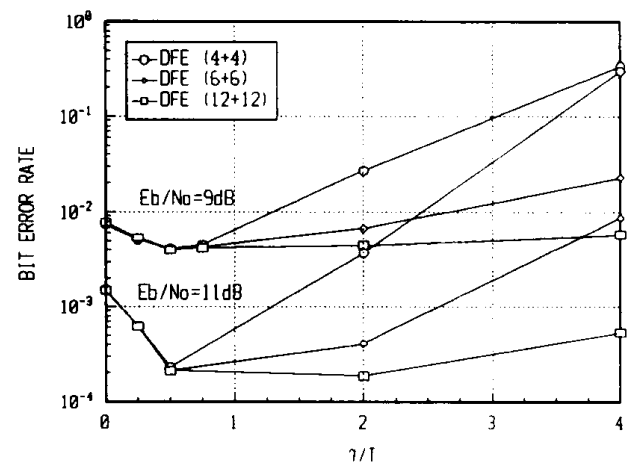

Fig. 3. BER for $K=1$ as a function of $\tau / T$ and with $E_{b} / N_{0}$ and DFE structure as parameters. $\left(f_{d} T=4.010^{-4}\right.$, 20 hopping frequencies)

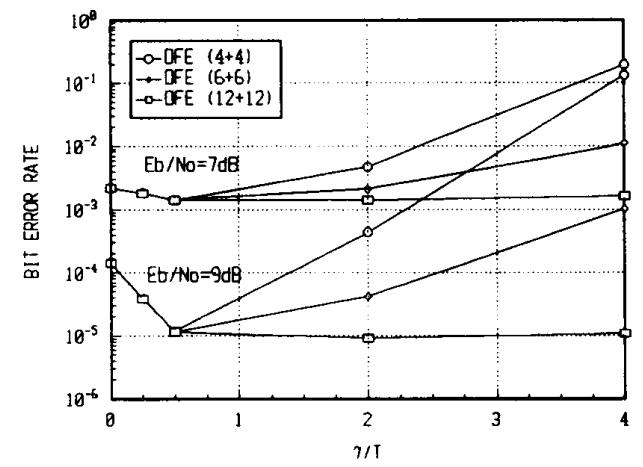

Fig. 4. BER for $K=2$ as a function of $\tau / T$ and with $E_{b} / N_{0}$ and DFE structure as parameters. $\left(f_{d} T=4.010^{-4}\right.$, 20 hopping frequencies).

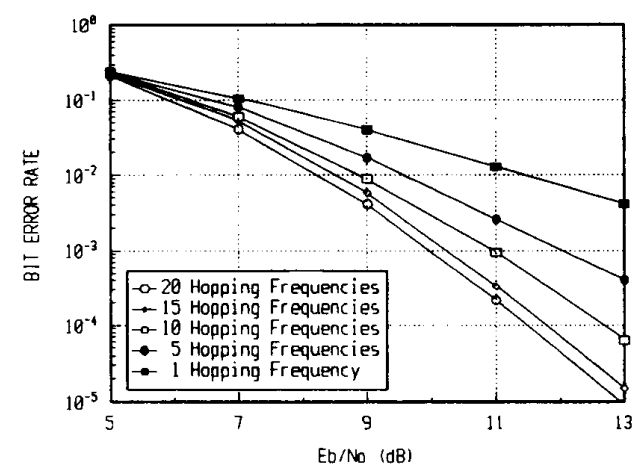

Fig. 5. BER performance for $K=1$ with the number of hopping frequencies as parameter. $(\tau / T=.5$, $f_{d} T=4.010^{-4}$, DFE $\left.(6+6)\right)$

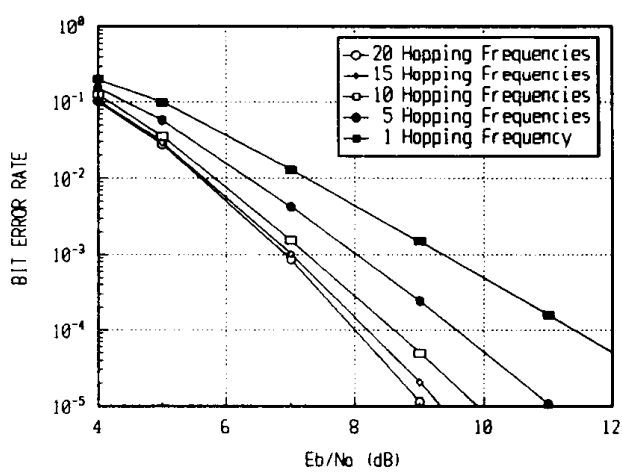

Fig. 6. BER performance for $K=2$ with the number of hopping frequencies as parameter. $(\tau / T=.5$, $f_{d} T=4.010^{-4}$, DFE $\left.(6+6)\right)$ 